\title{
Performance of a New Enhanced Topological Decision-Rule Map-Matching Algorithm for Transportation Applications
}

\author{
C. Blazquez $\star^{1}$, P. Miranda ${ }^{2}$, A. Ponce ${ }^{3}$ \\ ${ }^{1,3}$ Departamento Ciencias de la Ingeniería \\ Universidad Andres Bello \\ Santiago, Chile \\ *cblazquez@unab.cl \\ 2 Escuela de Ingeniería Industrial \\ Pontificia Universidad Catolica de Valparaiso \\ Valparaíso, Chile
}

\begin{abstract}
Map-matching problems arise in numerous transportation-related applications when spatial data is collected using inaccurate GPS technology and integrated with a flawed digital roadway map in a GIS environment. This paper presents a new enhanced post-processing topological decision-rule map-matching algorithm in order to address relevant special cases that occur in the spatial mismatch resolution. The proposed map-matching algorithm includes simple algorithmic improvements: dynamic buffer that varies its size to snap GPS data points to at least one roadway centerline; a comparison between vehicle heading measurements and associated roadway centerline direction; and a new design of the sequence of steps in the algorithm architecture. The original and new versions of the algorithm were tested on different spatial data qualities collected in Canada and United States. Although both versions satisfactorily resolve complex spatial ambiguities, the comparative and statistical analysis indicates that the new algorithm with the simple algorithmic improvements outperformed the original version of the map-matching algorithm.
\end{abstract}

Keywords: spatial data, global positioning systems, intelligent transportation systems, map-matching algorithm.

\section{RESUMEN}

El problema de la ambigüedad espacial ocurre en varias aplicaciones relacionadas con transporte, específicamente cuando existe inexactitud en los datos espaciales capturados con tecnología GPS o cuando son integrados con un mapa digital que posee errores en un ambiente SIG. Este artículo presenta un algoritmo nuevo y mejorado basado en reglas de decisión que es capaz de resolver casos especiales relevantes en modo post-proceso. El algoritmo propuesto incluye las siguientes mejoras algorítmicas: un área de búsqueda dinámica que varía su tamaño para asociar puntos GPS a al menos un eje de calzada, una comparación entre el rumbo del vehículo y la dirección del eje de calzada asignada, y un nuevo diseño de la secuencia de pasos del algoritmo. Tanto el algoritmo original como el propuesto fueron examinados con datos espaciales de diferentes calidades capturados en Canadá y Estados Unidos. Aunque ambas versiones resuelven satisfactoriamente el problema de ambigüedad espacial, el análisis comparativo y estadístico indica que la nueva versión del algoritmo con las mejoras algorítmicas entrega resultados superiores a la versión original del algoritmo.

\section{Introduction}

Global Positioning System (GPS) integrated with Geographic Information Systems (GIS) are part of the main components of innovative, advanced technology applied in intelligent transportation systems, in order to yield a more efficient transportation system, less congested, safer, and less polluting [1] [2] [3] [4] [5]. When integrating GPS data points and roadway network data in a GIS environment, spatial analysis software projects GPS data points orthogonally onto nearest roadways by calculating the minimum distance between each GPS data point and each roadway representation. This process is called "snapping". A map-matching problem occurs when a GPS data point is snapped to an incorrect roadway centerline because of lack of accuracy in the digital cartography, the GPS measurements, or both. Consequently, the location of events, incidents, or moving vehicles is assigned to incorrect roadway segments and, thus, affecting any subsequent process of usage, computation, evaluation, analysis, planning, and decision making. 
These spatial ambiguities arise in many transportation applications producing significant potential consequences [6] [7] [8] [9] [10]. For example, charging errors in automatic road toll systems due to uncorrected map-matching problems are an unacceptable outcome. These systems rely on efficient and robust map-matching techniques in the time, distance, and location computation for correct road use charging based on the total mileage driven per vehicle [11] [12] [13]. In other transportation applications in the United States, state and county transportation decision makers employ performance measures and decision management tools for winter maintenance operations. These require correct map-matched GPS data to compute accurate accumulated distances per patrol section [14]. Papinski et al. [15] identified the need of efficient post-processing map-matching algorithms for accurately determining user route choices. GPS data provides trip information on exact start and end trip times along with the routes traveled. If this data is coupled with diary data, then new insights are obtained about the trip planning behavior and route-choice decision making. Yoon et al. [16] described a research to quantify the Atlanta regional transit bus speed and acceleration rates that are applied as inputs to load-based modal mobile source emissions models. Through a mapmatching process, speed data were associated with different roadway facility types, and subsequently, emission levels. Emergency response, pickup/delivery services, travel behavior studies, and solid waste collection systems are other examples of transportation applications, where GPS data are collected and stored for later retrieval, processing, and analysis for strategic decision making and route planning.

Several approaches have been proposed in the literature to solve the map-matching problem. These methodologies have different levels of complexity ranging from simple search techniques to advanced and sophisticated inference, filtering, and mathematical modeling approaches such as Kalman filters, fuzzy logic, and Bayesian statistics [1] [17] [18] [19]. Recently, topological map-matching algorithms are more inclined to be employed in solving spatial mismatches due to the rapid and simple implementation of these algorithms [20].
Blazquez and Vonderohe [17] described a postprocessing topological decision-rule mapmatching algorithm to determine the correct route on which a vehicle traveled, based on vehicle speed, network topology, and turn restrictions. The algorithm successfully solves the map-matching problem, as shown in [21]. However, certain issues remain to be addressed and analyzed in the map-matching resolution.

The objective of this paper is to propose a new enhanced topological map-matching algorithm that yields an increased success level in solving spatial mismatches when compared to the original algorithm, without the need of additional complex computations. In this study, the performance of the proposed algorithm is assessed by comparing average percentages for solved and unsolved spatial ambiguities. A statistical analysis is achieved to explore the significance of each improvement with respect to the original algorithm results.

\section{Original topological decision-rule map- matching algorithm}

The original decision-rule map-matching algorithm selects all roadways within a buffer around each analyzed GPS data point and orthogonally projects it to the closest roadway by determining the minimum perpendicular distance between this point and each roadway centerline. Subsequently, Dijkstra's algorithm is employed to solve the shortest path problem between previously snapped and current snapped points using network topology and turn restrictions, as shown in Figure 1 . In this example, data points 1 and 2 are snapped to the closest roadway (i.e., Ramp 2) contained within the buffers around the data points.

The shortest distance traveled $D$ (displayed with a bold arrow) is obtained between the two snapped GPS data points (S1 and S2), shown as squares. The travel speed $s$ between these two snapped GPS points is computed using Equation 1, where $D$ is the length of the shortest path and $\left(t_{2}-t_{1}\right)$ is the difference in time stamps for the points. This speed is compared to the average of the speeds $\left(v_{1}\right.$ and $\left.v_{2}\right)$ at the data points collected by the vehicle while traveling, using Equation 2. If the average recorded speed $v$ is within an equally distributed speed range $r_{s}$ around travel speed $s$ 
(See Equation 3), then the obtained shortest path is viable and the snapped locations for points 1 and 2 are accepted as correct. If the path is rejected, then data points are snapped to alternative roadway centerlines contained within their buffers, shortest paths are recalculated, and speeds are compared once again. If no other roadway centerlines exist within the buffers or no feasible paths are obtained, then the algorithm tests for feasible paths between preceding and subsequent snapped data points.

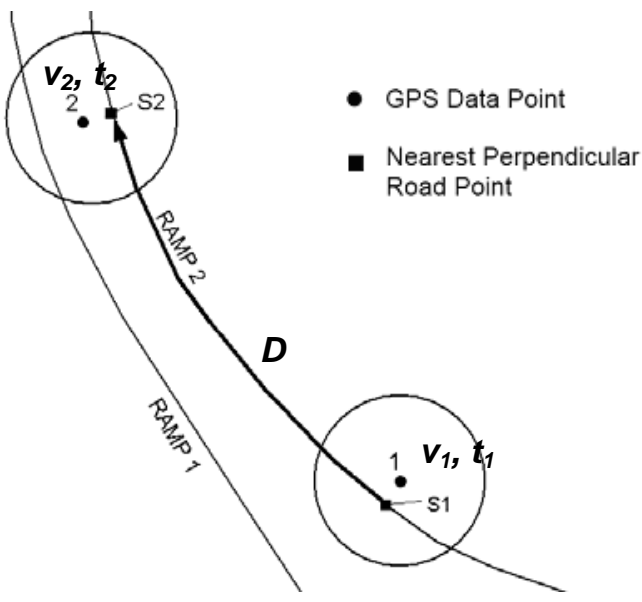

Figure 1. Example of snapping process to the correct roadway for two GPS data points.

$$
\begin{aligned}
& s=\frac{D}{\left(t_{2}-t_{1}\right)} \\
& v=\frac{v_{1}+v_{2}}{2} \\
& v \in\left[s \pm \frac{r_{s}}{2}\right]
\end{aligned}
$$

The flow chart illustrated in Figure 2 shows the methodology for the shortest path calculation between neighboring data points and alternative data point snapping locations until a feasible path is obtained or five consecutive data points ( $\mathrm{Ki}-2$, $\mathrm{Ki}-1, \mathrm{Ki}, \mathrm{Kj}$, and $\mathrm{Kj}+1$ ) are examined. If a feasible path between snapped points $\mathrm{Ki}$ and $\mathrm{Kj}$ is obtained due to a successful speed comparison, then the spatial ambiguity is solved and the algorithm ends.

Otherwise, the algorithm computes the shortest path between points $\mathrm{Kj}$ and $\mathrm{Kj}+1$. If the path between this pair of points is not viable, then the algorithm performs a resnapping process to alternative roadway centerlines within the buffer of point $\mathrm{Kj}(\mathrm{Alt} \mathrm{Kj})$. If the resnapping of point $\mathrm{Kj}$ is successful, then the existence of feasible paths between this newly snapped point (Ks), and preceding and succeeding neighboring snapped points Ks-1 and Ks+1 is verified. If the latter fails, then alternative snapping locations are searched within the buffer of point $\mathrm{Ks}$, and shortest paths between neighboring points are tested again. If no feasible snapping alternatives exist for data point $\mathrm{Kj}$, then the algorithm performs additional testing, looking ahead by examining the shortest paths between snapped points $\mathrm{Ki}$ and $\mathrm{Kj}+1$, points $\mathrm{Ki}-1$ and $\mathrm{Kj}$, points $\mathrm{Ki}-2$ and $\mathrm{Kj}$, and alternative roadways contained within the buffers of points $\mathrm{Ki}$ and $\mathrm{Ki}-1$. If a feasible path between any of these pairs of points is obtained, then intermediate points are forced to snap to the roadway centerline along the computed path solving the spatial ambiguity and terminating the algorithm.

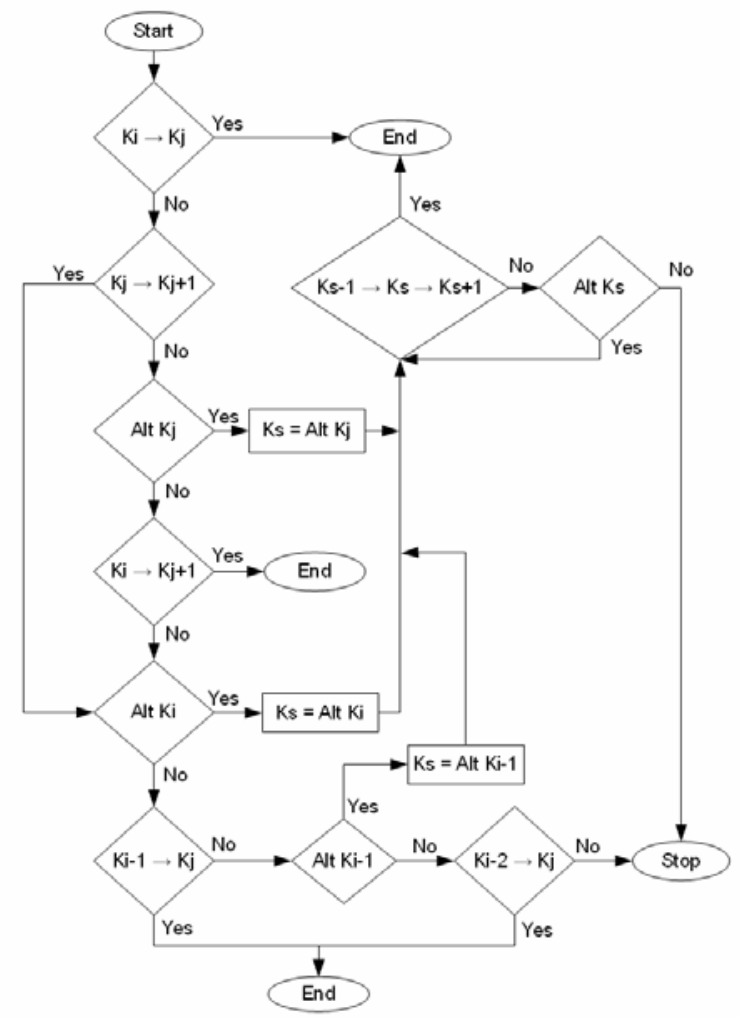

Figure 2. Flow chart for the original step sequence of the algorithm. 


\section{Typical unsolved map-matching problems}

Although the original algorithm successfully solves most of the spatial mismatches [21], there are three unsolved cases that still require attention. The first two cases occur as a result from inadequate parameter values and design of the algorithm, and the third case arises when the vehicle travels on bidirectional roads. Examples are presented for each case highlighting the limitations and possible improvements.

Case I: Unsnapped data points due to constant buffer size The original algorithm employs a constant buffer size around each data point to select at least one road candidate during the snapping process. If the buffer size is too small, then data points do not snap to any road. Figure 3 shows an example in which three successive points fail to snap to Interstate 39 . This problem is solved if the buffer is increased dynamically as the algorithm is executed until one or more roadway centerlines are contained within the buffer.

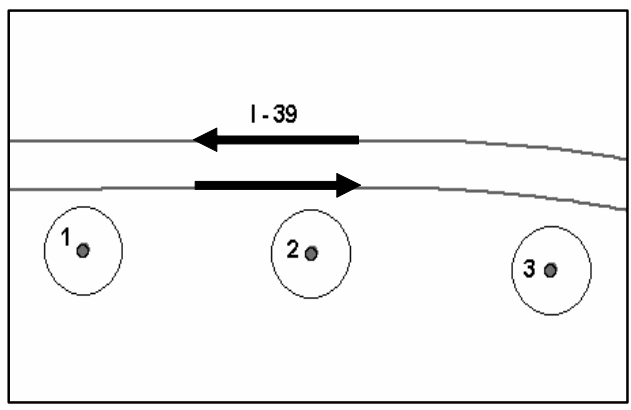

Figure 3. Example of three consecutive unsnapped GPS data points.

Case II: Incorrect snapping due to inadequate algorithmic design The design of the original algorithm follows an inappropriate step sequence, which performs feasible path verifications only with end points $\mathrm{Kj}$ or $\mathrm{Kj}+1$, and examines feasible alternative locations within buffers solely for points $\mathrm{Ki}-1, \mathrm{Ki}$, and $\mathrm{Kj}$. In addition, the algorithm limits the map-matching resolution to at most five number of consecutive data points. As a consequence, points are snapped incorrectly without solving the spatial ambiguity. For example, Figure 4 illustrates a vehicle path along an interchange in Wisconsin, in which points 2 through 5 are incorrectly snapped to the ramp since no feasible path was obtained between data points 5 and 6 given their current snapped positions (Ramp and Interstate 39, respectively). This map-matching problem is solved if the number of consecutive points is increased to six, and a feasible path is determined along Interstate 39 between snapped points 1 and 6 . Subsequently, the spatial mismatch is solved and data points 2 through 5 are forced to snap correctly along the path on Interstate 39 .

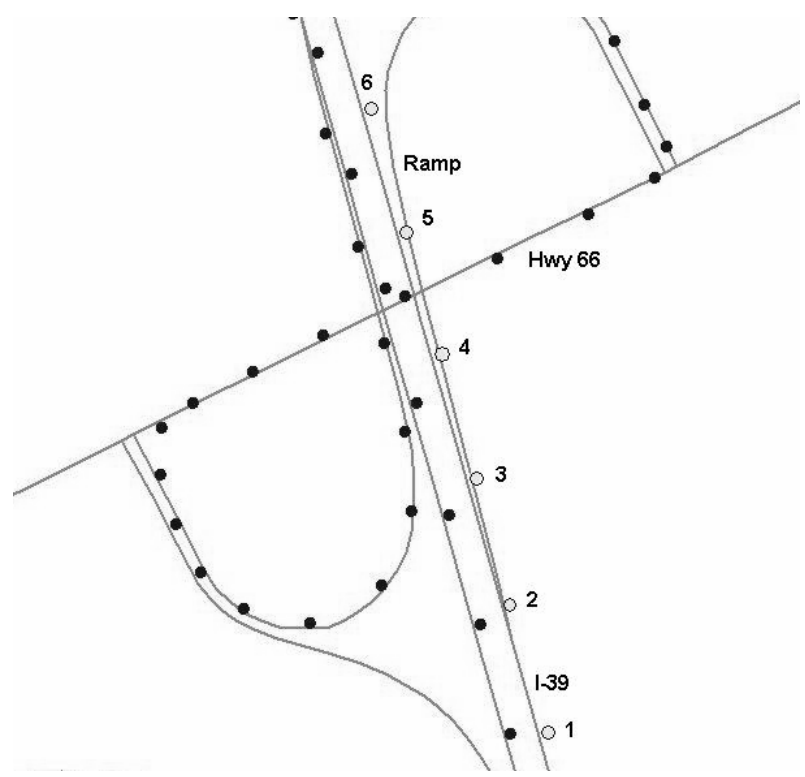

Figure 4. Example of a map-matching problem not solved because of insufficient number of consecutive data points.

Case III: Incorrect snapping at converging, diverging, or at-grade bidirectional roads Figure 5 depicts an example of a spatial ambiguity that occurs when a vehicle travels on bidirectional roads. After executing the map-matching algorithm, a feasible route between points 2 and 3 (shown with bold arrows) is computed as a result of the successful speed comparison. However, points 0,1 , and 2 remain incorrectly snapped to Highway 51 instead of Highway 16. Figure 6 shows another example of a spatial mismatch that occurs at at-grade intersections when roads are bidirectional. Data point 2, located close to the intersection of Roads 1 and 2, snaps erroneously to Road 2 instead of Road 1. Feasible shortest 
paths are computed between data points 1 and 2, and subsequently, between data points 2 and 3 . The map-matching algorithm successfully compared the recorded and calculated speeds, and incorrectly accepted the snapping location of point 2 as correct. Vehicle heading data is an algorithmic parameter commonly used as a strategy to solve the type of spatial mismatches presented in Figures 5 and 6 [19] [22]. Thus, if both vehicle speeds and headings are compared, then the correct snapped GPS positions are determined solving the spatial ambiguities.

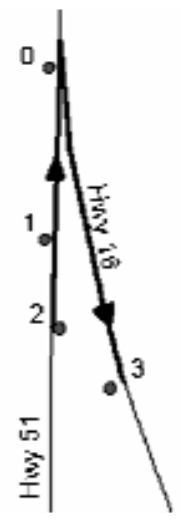

Figure 5. Example of a bidirectional diverging road ambiguity not resolved.

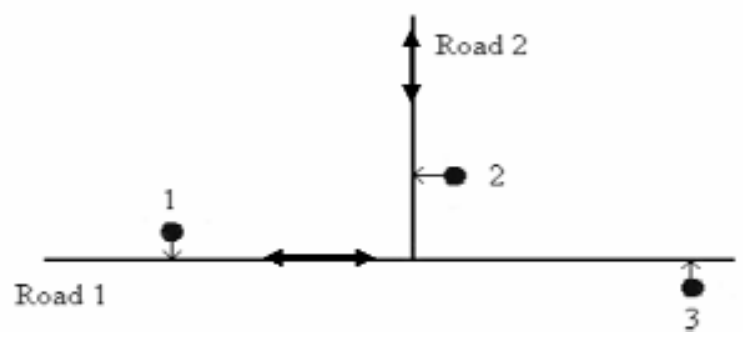

Figure 6. Example of at-grade intersection ambiguity not resolved.

\section{Algorithmic enhancements}

Given the unsolved map-matching problems identified in the previous section, this study presents a new algorithm with simple algorithmic improvements: a) a dynamic buffer, b) a comparison between vehicle heading, and c) a new enhanced design in the step sequence of the algorithm that employs any given number of consecutive data points. Figure 7 presents an overall flow diagram of the new proposed algorithm indicating with dashed lines each of the algorithmic enhancements.

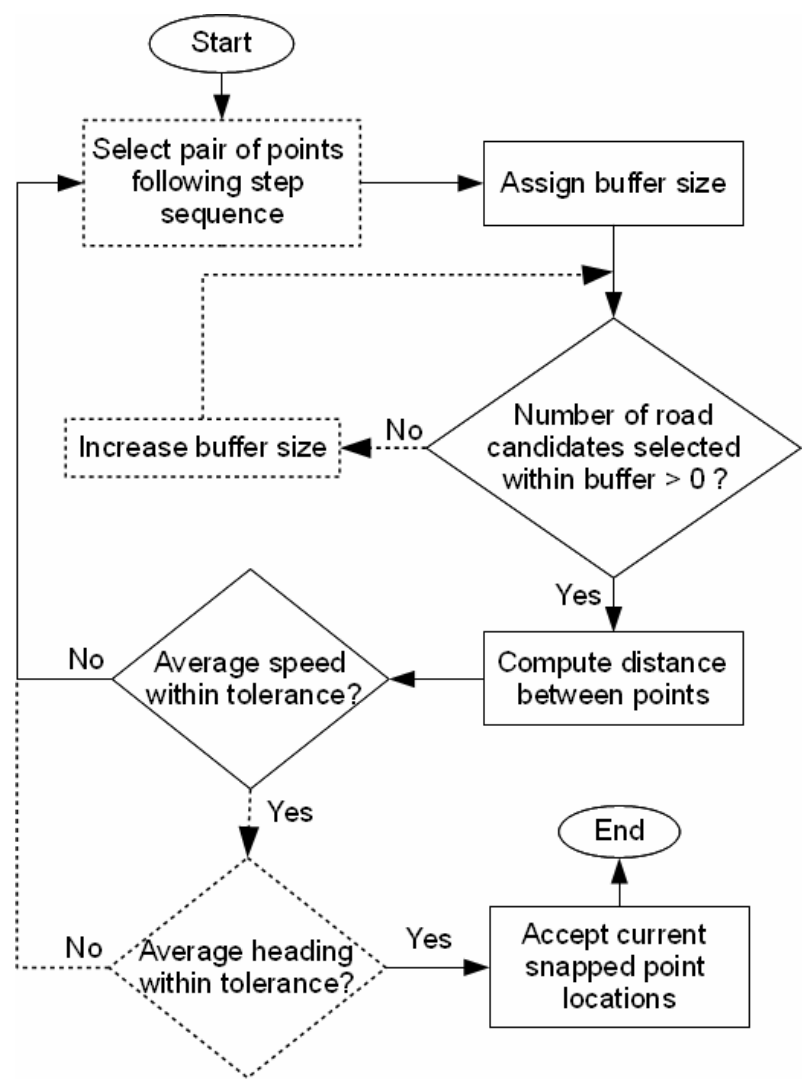

Figure 7: Overall flow diagram of the map-matching algorithm.

a) Dynamic Buffer

The new enhanced map-matching algorithm increments the buffer size proportionally until one or more roadway centerline candidates are contained within the buffer. Hence, at least one roadway centerline is tested as the buffer is incremented yielding a larger likelihood of selecting the correct roadway centerline.

b) Vehicle Heading

The proposed algorithm performs the speed evaluation, and consequently compares the vehicle heading with the roadway centerline direction defined by the azimuth at the snapped location. A 
tested path is viable if the azimuth $\phi$ of the snapped data point location on the roadway candidate is within a symmetrical heading range tolerance $q$ around the azimuth $\alpha$ of the vehicle traveling direction obtained from the GPS measurements, as shown in Equation 4.

$$
\phi \in\left[\alpha \pm \frac{q}{2}\right]
$$

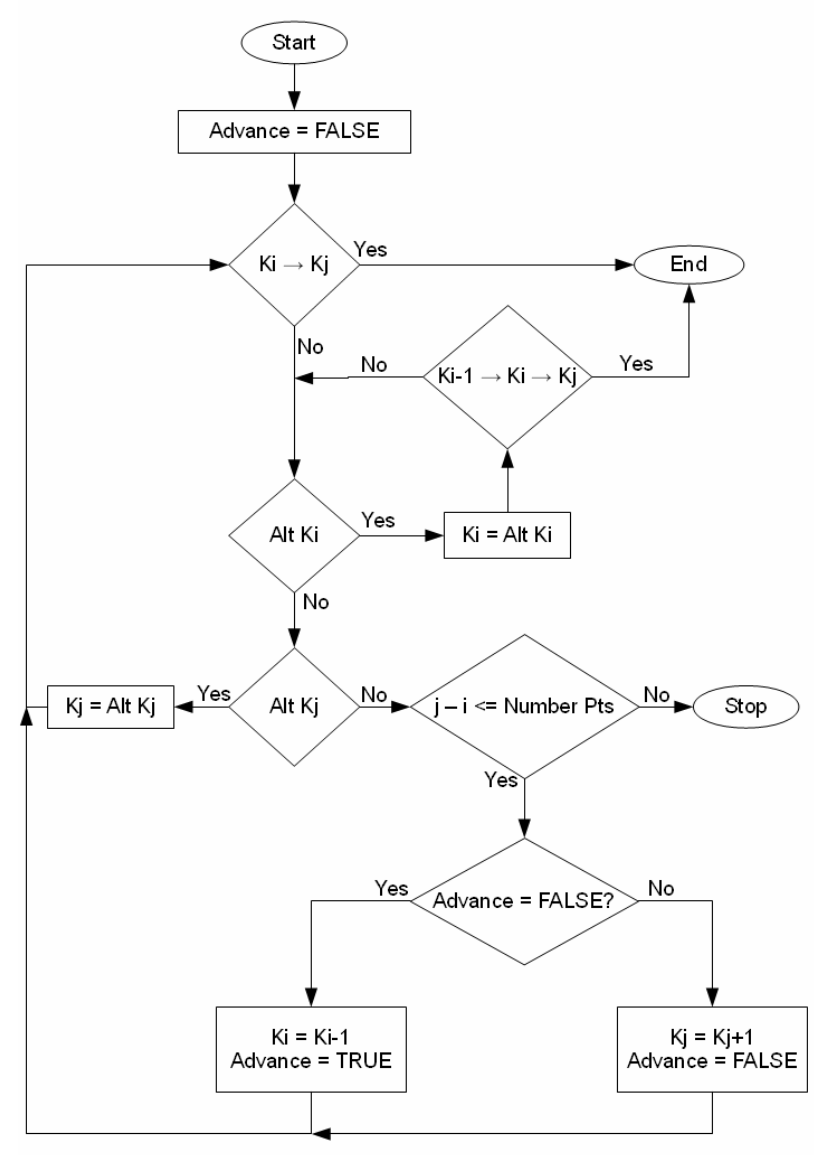

Figure 8. Flow diagram of the revised step sequence of the algorithm.

c) New Design of the Step Sequence

Figure 8 presents a new step sequence design in the map-matching algorithm, where a recursive procedure is followed until any predefined number of consecutive data points is explored. The new algorithm initiates by searching for alternative roadway centerlines contained within the buffer of point Ki when no feasible shortest path is obtained between snapped data points $\mathrm{Ki}$ and $\mathrm{Kj}$. If an alternative roadway candidate is found, then the algorithm tests for feasible paths between point $\mathrm{Ki}$ and immediate neighboring points $\mathrm{Ki}-1$ and $\mathrm{Kj}$. If no other roadway centerlines exist within the buffer of point $\mathrm{Ki}$, then data point $\mathrm{Kj}$ is snapped to alternative roadway candidates contained within its buffer, and shortest paths are recalculated. Additionally, the proposed algorithm employs the dummy Boolean variable "Advance" to broaden the test range either forward or backward on a one-by-one step basis until the map-matching problem is solved.

\section{Sample application}

The original and proposed map-matching algorithms were tested against different spatial sample data sets to determine the performance of the algorithmic improvements. Average percentages of solved and unsolved spatial ambiguities were calculated using 782 data points collected in Halifax, Canada and Portage County, Wisconsin in the United States. Note that heading measurement capabilities were not available during the data collection process in Portage County, no GPS signal quality such as number of satellites was provided, and none of the spatial data collected in this study employed an integrated Differential GPS (DGPS)/Dead Reckoning system.

The algorithmic enhancements were tested independently and combined with buffer size increments of 2 and 3 , heading tolerance values of $20^{\circ}$ and $30^{\circ}$, and 3,5 , and 7 consecutive data points. These improvements were compared to the original map-matching algorithm results with a buffer size of $20 \mathrm{~m}$, a speed range of $15 \mathrm{~km} / \mathrm{h}$, and 5 consecutive data points.

\subsection{Independent comparative analysis}

Figure 9 illustrates average percentages of solved spatial ambiguities for 11 vehicle routes in Halifax and Portage County after applying the original algorithm and each algorithmic enhancement independently. Parameter values for every improvement are shown on top of each column in the chart. Average percentages of solved spatial mismatches remained constant throughout the 


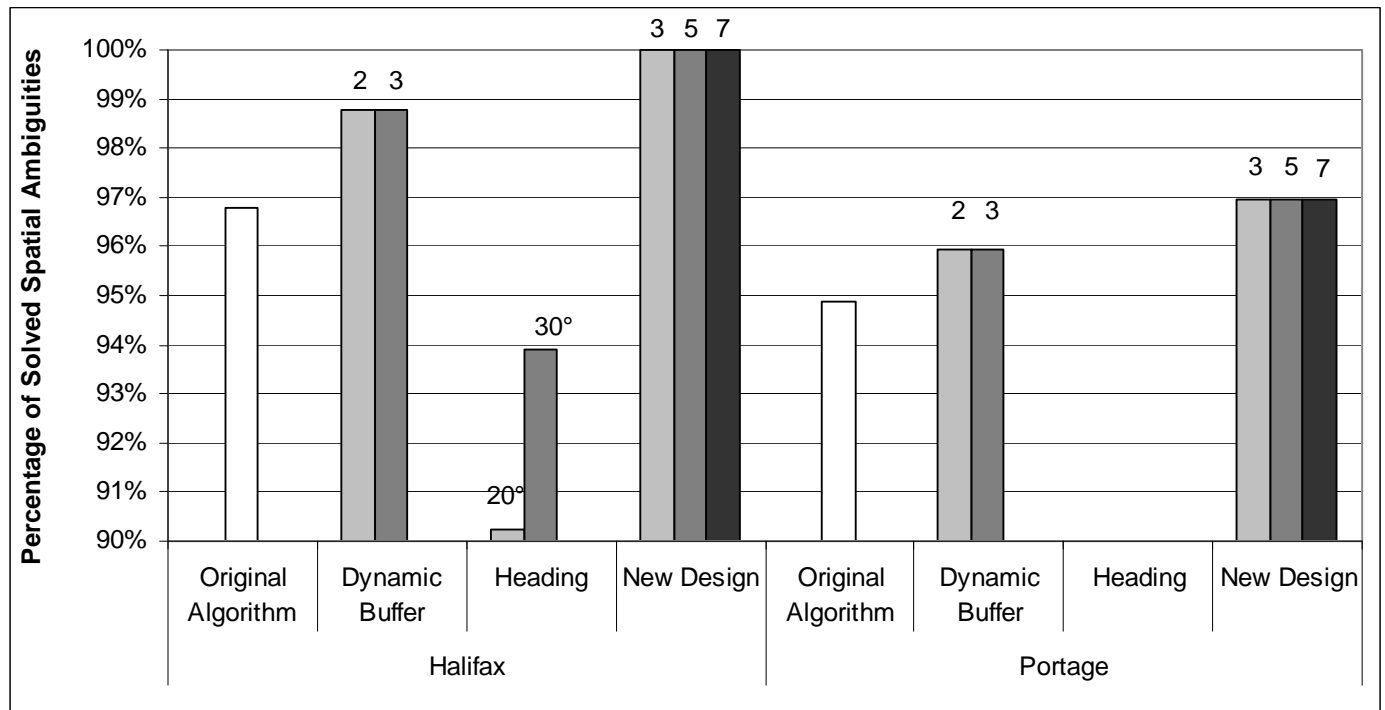

Figure 9. Average percentages of solved spatial ambiguities using original and enhanced algorithms for 11 vehicle routes in Halifax and Portage County.

analysis after applying the original algorithm since they do not depend on the parameter values of the new enhanced algorithm.

When comparing the dynamic buffer enhancement to the original algorithm, a $2.1 \%$ and $1.1 \%$ increase in the algorithmic performance was obtained for Halifax and Portage County routes, respectively. No improvement was observed in solving the mapmatching problem as the buffer size was increased from 2 to 3 , since points are snapped to incorrect roads that are selected within a larger buffer.

The map-matching results depend significantly on the quality of the vehicle heading measurements. These are influenced negatively particularly when vehicles travel at low speeds or are idling [20]. For example, Halifax vehicle routes include slow traveling speeds in a dense urban environment producing poor quality in the heading measurements, thus the heading comparison yields lower solved case percentages than the original algorithm results.

The new step sequence design of the algorithm outperformed the original algorithm for all Halifax routes obtaining $100 \%$ of the map-matching problems solved even when only three consecutive points were utilized. No additional improvements in the percentage of solved spatial mismatches were perceived when using more than three consecutive points. Similarly, three consecutive points are adequate in solving spatial mismatches that occurred with the Portage County tested data. Overall, these results indicate that the new step sequence of the proposed map-matching algorithm contributes to solving a larger number of spatial ambiguities than the dynamic buffer and the heading range enhancements applied independently.

Table 1 shows the average number of solved and unsolved spatial ambiguities after applying the original and independent algorithmic enhancements for all routes. The average number of unsolved points includes cases 1, 2, and 3 (as explained in Section 3), and other undetermined map-matching cases. Notice that the heading comparison improvement was achieved only with Halifax routes, and thus, the inferior total number of data points tested.

\subsection{Combined comparative analysis}

Figure 10 presents variations in the average percentage of solved spatial ambiguities for 11 vehicle routes in Halifax, and Portage County after applying the original algorithm and combined 
algorithmic enhancements, where "a" is the dynamic buffer, " $b$ " is the heading range, and " $c$ " is the new algorithmic design. Solved spatial mismatch percentages remained constant throughout the analysis after implementing the original algorithm.

\begin{tabular}{|c|c|c|c|c|c|c|c|}
\hline \multirow{2}{*}{\multicolumn{2}{|c|}{ Algorithm }} & \multirow{2}{*}{$\begin{array}{c}\text { Total } \\
\text { number } \\
\text { of } \\
\text { points }\end{array}$} & \multirow{2}{*}{$\begin{array}{c}\text { Number } \\
\text { of solved } \\
\text { points }\end{array}$} & \multicolumn{4}{|c|}{ Number of unsolved points } \\
\hline & & & & Case 1 & Case 2 & Case 3 & Other \\
\hline \multicolumn{8}{|c|}{ Original } \\
\hline & & 782 & 750 & 2 & 15 & 15 & 0 \\
\hline \multicolumn{8}{|c|}{ Independent enhancements } \\
\hline \multirow{2}{*}{$a$} & 2 & 782 & 761 & 1 & 10 & 10 & 0 \\
\hline & 3 & 782 & 760 & 1 & 10 & 11 & 0 \\
\hline \multirow[b]{2}{*}{ b } & $20^{\circ}$ & 424 & 382 & 0 & 8 & 7 & 27 \\
\hline & $30^{\circ}$ & 424 & 398 & 0 & 3 & 3 & 20 \\
\hline \multirow{3}{*}{ C } & 3 points & 782 & 770 & 2 & 4 & 6 & 0 \\
\hline & 5 points & 782 & 770 & 2 & 4 & 6 & 0 \\
\hline & 7 points & 782 & 770 & 2 & 4 & 7 & 0 \\
\hline
\end{tabular}

Table 1. Average number of solved and unsolved data points for original and independent enhancements of the algorithm.

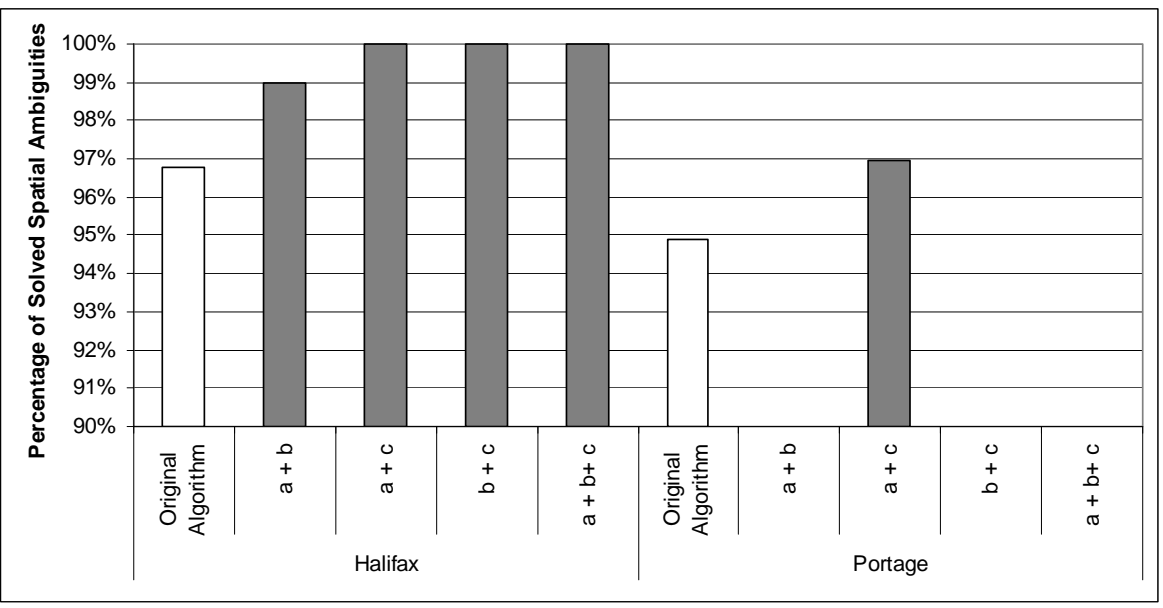

Figure 10. Average percentages of solved spatial ambiguities for original algorithm and combined algorithmic enhancements in Halifax and Portage County.

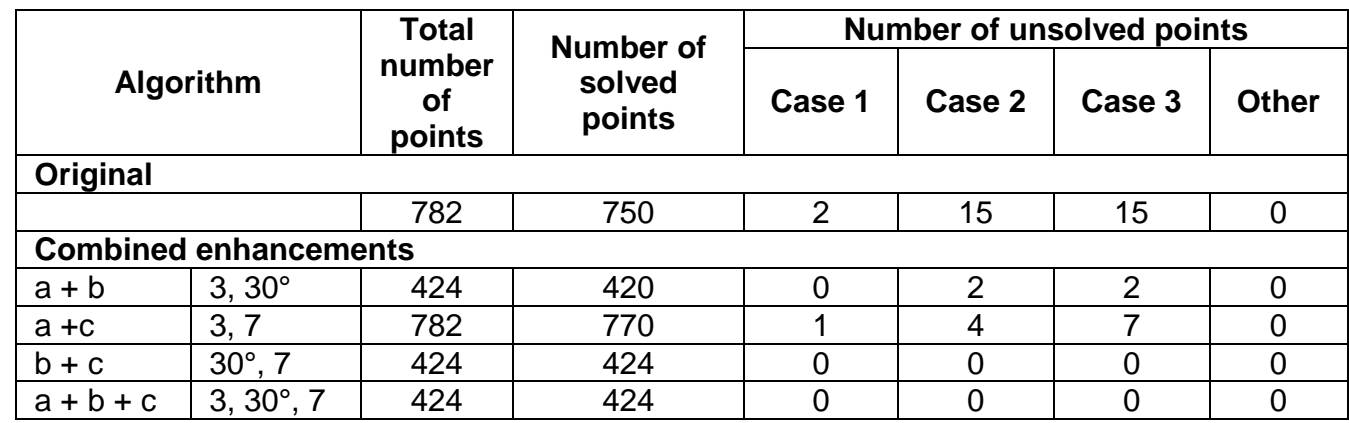

Table 2. Average number of solved and unsolved data points for original and combined enhancements of the algorithm. 
Improved results in solving the map-matching problem were obtained with all enhanced combinations when compared to the original algorithm. The addition of the dynamic buffer (a) and/or the new algorithmic design (c) to the heading range enhancement is able to enrich the results of the independent comparative analysis for Halifax data set. The percentage of solved cases for Portage County routes perceived an increase of $2.1 \%$ with respect to the original algorithm results when implementing the dynamic buffer/revised algorithm $(a+c)$ combination. Table 2 presents the average number of solved and unsolved spatial ambiguities after applying the original algorithm and best combinations for the enhancements with a buffer increment of 2 , heading range of $30^{\circ}$, and 7 consecutive data points.

\section{Statistical analysis}

This section provides a basic statistical analysis, in order to support the advantages of the new proposed methodology for the map-matching algorithm. This statistical analysis was applied to 1,918 data points collected for 24 routes in Halifax and Portage County. As a result from the sample analysis, the heading-based improvement is not considered due to the poor results obtained with this modification. Therefore, the algorithm enhancements include the dynamic buffer $(a=2$ and $a=3)$ and the new step sequence design of the algorithm $(c=3, c=5$, and $c=7)$.

Table 3 presents the aggregated statistical results from the comparison between the original algorithm and the proposed enhanced algorithm. This table shows the average percentage, the variance (depicted in parenthesis), and the respective $t$ statistic for solved and three types of unsolved cases previously identified. In order to evaluate statistic significance on the observed differences, the $t$ statistic was computed for each case by comparing the results of the enhanced algorithm implementations and the original. Naturally, the $t$ statistic was not computed for the original algorithm.

The $t$-statistic for the solved cases was calculated by subtracting the results of the enhanced algorithm from the original results, whereas the $t$ statistic for the unsolved cases was obtained by computing the difference between the results of the original algorithm and the enhanced algorithm presenting positive values. In both cases, a hypothesis testing was developed for the difference between two means. For example, the mean average of the solved cases for each algorithmic enhancement and the original algorithm were compared. The null hypothesis $\left(\mathrm{H}_{0}\right)$ denotes that both means are equal, and the alternative hypothesis $\left(\mathrm{H}_{1}\right)$ indicates that the mean of the solved cases is greater for the enhanced algorithm than for the original version of the algorithm. If the t-student is greater than a critical value, $\mathrm{H}_{0}$ is rejected and there is significant difference between the means (shown in bold). On the contrary, $\mathrm{H}_{0}$ is accepted and there is no difference between the means.

Considering a significance level of $5 \%$, the critical value of the hypothesis test for the mean difference is 2,013. Based on this critical value, the independent implementation of dynamic buffer and new step sequence design are not statistically better than the original algorithm. However, when both improvements are combined $(a+c)$, enhanced results are observed, and thus, outperforming statistically the original algorithm for values $\mathrm{a}=2$ and $\mathrm{c}=7$.

Regarding the results for each type of unsolved cases (I, II, and III), the best combination is better statistically than the original algorithm only for the unsolved case I. However, close statistical significance is perceived for the unsolved case II, when employing a 90\% confidence level and a critical value of 1,679 .

The best combination of the new enhanced algorithm $(\mathrm{a}=2$ and $\mathrm{c}=7)$ presents better results for all 24 routes by increasing the percentage of solved spatial ambiguities, and reducing the percentage of each unsolved case. The enhanced algorithm presented the worst results for only one route, when the dynamic buffer was amplified by 2 and 3 independently $(a=2$ and $a=3)$, and also for all combinations using a dynamic buffer of 3 (i.e., $(3,3)$; $(3,5)$; and $(3,7))$. This may occurred when the buffer size is too large for the sample data set and the improved algorithm snaps some data points incorrectly. For all the remaining algorithmic combinations, the enhanced algorithm always statistically outperformed the original algorithm with all routes. 


\begin{tabular}{|c|c|c|c|c|c|c|}
\hline \multirow{2}{*}{\multicolumn{2}{|c|}{ Algorithm }} & \multirow{2}{*}{ Solved Cases } & \multicolumn{4}{|c|}{ Unsolved Cases } \\
\hline & & & Case I & Case II & Case III & Total \\
\hline \multirow{2}{*}{\multicolumn{2}{|c|}{ Original }} & $87,74 \%$ & $1,60 \%$ & $3,47 \%$ & $7,19 \%$ & $12,26 \%$ \\
\hline & & $(0,0146)$ & $(0,0007)$ & $(0,0049)$ & $(0,0100)$ & $(0,0146)$ \\
\hline \multicolumn{7}{|c|}{ Independent Enhancements } \\
\hline \multirow{6}{*}{ a } & & $90,69 \%$ & $1,16 \%$ & $2,38 \%$ & $5,77 \%$ & $9,31 \%$ \\
\hline & 2 & $(0,0099)$ & $(0,0005)$ & $(0,0016)$ & $(0,0074)$ & $(0,0099)$ \\
\hline & & 0,922 & 0,632 & 0,657 & 0,526 & 0,922 \\
\hline & & $90,75 \%$ & $1,15 \%$ & $2,26 \%$ & $5,84 \%$ & $9,25 \%$ \\
\hline & 3 & $(0,0108)$ & $(0,0005)$ & $(0,0014)$ & $(0,0075)$ & $(0,0108)$ \\
\hline & & 0,925 & 0,635 & 0,745 & 0,500 & 0,925 \\
\hline \multirow{9}{*}{ c } & & $92,41 \%$ & $1,08 \%$ & $1,80 \%$ & $4,71 \%$ & $7,59 \%$ \\
\hline & 3 & $(0,0079)$ & $(0,0003)$ & $(0,0012)$ & $(0,0059)$ & $(0,0079)$ \\
\hline & & 1,524 & 0,812 & 1,045 & 0,963 & 1,524 \\
\hline & & $93,29 \%$ & $0,86 \%$ & $1,77 \%$ & $4,08 \%$ & $6,71 \%$ \\
\hline & 5 & $(0,0059)$ & $(0,0002)$ & $(0,0011)$ & $(0,0043)$ & $(0,0059)$ \\
\hline & & 1,899 & 1,202 & 1,071 & 1,276 & 1,899 \\
\hline & & $93,50 \%$ & $0,86 \%$ & $1,56 \%$ & $4,08 \%$ & $6,50 \%$ \\
\hline & 7 & $(0,0055)$ & $(0,0002)$ & $(0,0011)$ & $(0,0043)$ & $(0,0055)$ \\
\hline & & 1,988 & 1,202 & 1,204 & 1,276 & 1,988 \\
\hline \multicolumn{7}{|c|}{ Combined Enhancements } \\
\hline \multirow{18}{*}{$a+c$} & & $92,85 \%$ & $0,66 \%$ & $1,65 \%$ & $4,83 \%$ & $7,15 \%$ \\
\hline & 2,3 & $(0,0065)$ & $(0,0002)$ & $(0,0011)$ & $(0,0056)$ & $(0,0065)$ \\
\hline & & 1,723 & 1,535 & 1,146 & 0,923 & 1,723 \\
\hline & & $93,79 \%$ & $0,66 \%$ & $0,92 \%$ & $4,63 \%$ & $6,21 \%$ \\
\hline & 2,5 & $(0,0050)$ & $(0,0002)$ & $(0,0006)$ & $(0,0048)$ & $(0,0050)$ \\
\hline & & 2,118 & 1,535 & 1,683 & 1,031 & 2,118 \\
\hline & & $94,09 \%$ & $0,37 \%$ & $0,83 \%$ & $4,71 \%$ & $5,91 \%$ \\
\hline & 2,7 & $(0,0053)$ & $(0,0001)$ & $(0,0006)$ & $(0,0050)$ & $(0,0053)$ \\
\hline & & 2,206 & 2,183 & 1,738 & 0,992 & 2,206 \\
\hline & & $93,14 \%$ & $0,44 \%$ & $1,59 \%$ & $4,83 \%$ & $6,86 \%$ \\
\hline & 3,3 & $(0,0070)$ & $(0,0001)$ & $(0,0013)$ & $(0,0056)$ & $(0,0070)$ \\
\hline & & 1,797 & 2,051 & 1,166 & 0,923 & 1,797 \\
\hline & & $93,82 \%$ & $0,44 \%$ & $1,04 \%$ & $4,71 \%$ & $6,18 \%$ \\
\hline & 3,5 & $(0,0055)$ & $(0,0001)$ & $(0,0008)$ & $(0,0050)$ & $(0,0055)$ \\
\hline & & 2,101 & 2,051 & 1,576 & 0,992 & 2,101 \\
\hline & & $93,88 \%$ & $0,37 \%$ & $1,04 \%$ & $4,71 \%$ & $6,12 \%$ \\
\hline & 3,7 & $(0,0054)$ & $(0,0001)$ & $(0,0008)$ & $(0,0050)$ & $(0,0054)$ \\
\hline & & 2,131 & 2,183 & 1,576 & 0,992 & 2,131 \\
\hline
\end{tabular}

Table 3. Summary of statistic results. 


\section{Conclusions}

This paper proposes a new enhanced topological decision-rule map-matching algorithm to solve particular unsolved cases that occur in the mapmatching problem resolution. This algorithm includes the following key elements: a) a dynamic buffer that accommodates by increasing its size to contain at least one roadway centerline; b) a vehicle heading and roadway centerline azimuth comparison; and c) a new step sequence design of the algorithm.

The original and proposed versions of the algorithm were tested employing data collected in Halifax, Canada and Portage County, Wisconsin. The sample and statistical analysis presented in this paper confirms that the proposed map-matching algorithm with independent and combined enhancements was capable of outperforming the original map-matching algorithm for most of the tested spatial data. Among the three improvements of the proposed map-matching algorithm, the new step sequence design solved more incorrect snapped data points than the other improvements when implemented independently and combined for all routes. The percentage of solved spatial mismatches is increased if the dynamic buffer/revised algorithm combination is added to the heading range improvement, specifically when poor heading measurements are employed.

Future research is required to determine optimal parameter values for the new enhanced mapmatching algorithm using a theoretical statistical approach. Further research may involve the implementation of variable speed and heading range tolerance values when traveling in different operational environments.

\section{Acknowledgements}

Financial support from the Chilean National Fund for Scientific and Technological Development (FONDECYT 1070386) is acknowledged. Anonymous review of this paper is also gratefully thanked.

\section{References}

[1] Y. Zhao, "Vehicle Location and Navigation Systems", Norwood, MA., Artech House, Inc., 1997.

[2] C. Drane, and C. Rizos, "Positioning Systems in ITS", Norwood, MA., Artech House, Inc., 1998.

[3] M. Chowdhury, and A. Sadek, "Fundamentals of Intelligent Transportation Systems Planning", Norwood, MA., Artech House, Inc., 2003.

[4] J. Sussman, "Perspective on Intelligent Transportation Systems", New York, NY., Springer Science \& Business Media, Inc., 2005.

[5] C. Nava-Fonseca, "Analysis of a Transport Network Based on Georeference System and Multicommodity Network Flow", Journal of Applied Research and Technology, Vol. 4, No. 1, pp. 50-58, 2006.

[6] V. Noronha, and M. Goodchild, "Map Accuracy and Location Expression In Transportation - Reality and Prospects", Transportation Research Part C, Vol. 8, pp. 53-69, 2000.

[7] E. Fekpe, T. Windholz, K. Beard, and K. Novak, "Quality and Accuracy of Positional in Transportation, Interim Report National Cooperative Highway Research Program 20-47(01)," Transportation Research Board of the National Research Council, Washington D.C., 2001.

[8] S. Hallmark, W. Schuman, S. Kadolph, and R. Souleyrette, "Integration of Spatial Point Features with Linear Referencing Methods", Journal of the Transportation Research Board, No. 1836, pp.102-110, 2003.

[9] S. Syed, and M. Cannon, "Fuzzy Logic Based-Mapmatching Algorithm for Vehicle Navigation System in Urban Canyons", in Institute of Navigation National Technical Meeting, San Diego, California, 2004. Available from: http://plan.geomatics.ucalgary.ca/papers/04ntmsalman_f ooters.pdf

[10] M. Quddus, R. Noland, and W. Ochieng, "Validation of Map-matching Algorithms using High Precision Positioning with GPS", The Journal of Navigation, Vol. 58, pp. 257-271, 2005. 
[11] P.-M. Cheng, M. Donath, X. Ma, S. Shekhar, and K. Buckeye, "Evaluation of Digital Maps for Road User Charging Applications", in 84th Annual Meeting of the Transportation Research Board, Washington D.C., 2005. Available from:

http://www.spatial.cs.umn.edu/Project/CTS/TRBDigitalMa p080104.pdf

[12] B. Grush, "The Case Against Map-Matching", European Journal of Navigation, Vol. 6, No.3, pp. 2-5, 2008.

[13] K. Sheridan, T. Dyjas, C. Botteron, J. Leclere, F. Dominic, and G. Marucco "Demands of Roads. An Assisted-GNSS Solution Uses the EGNOS Data Access Service", GPS World, Vol. 22, No. 3, 2011, pp. 28.

[14] A. Vonderohe, T. Adams, C. Blazquez, J. Maloney, and T. Martinelli, "GIS-Based Analysis of Intelligent Winter Maintenance Vehicle Data", in Transportation Research Circular E-C063: Sixth International Symposium on Snow Removal and Ice Control Technology, Spokane, Washington, 2004, pp. 348-360.

[15] D. Papinski, D. Scott, and S. Doherty, "Exploring the Route Choice Decision-Making Process: A Comparison of Planned and Observed Routes Obtained Using Person-Based GPS", Transportation Research Part F, Vol. 12, pp. 347-358, 2009.

[16] S. Yoon, H. Li, J. Jun, R. Guensler, J. Ogle, and M. Rodgers, "Methodology for Developing Transit Bus Speed-Acceleration Matrices for Load-Based Mobile Source Emissions Models", Journal of Transportation Research Board, No. 1941, pp. 26-33, 2005.

[17] C. Blazquez, and A. Vonderohe, "Simple MapMatching Algorithm Applied to Intelligent Winter Maintenance Vehicle Data", Journal of Transportation Research Board, No.1935, pp. 68-76, 2005.

[18] G. Taylor, and G. Blewitt, "Intelligent Positioning GISGPS Unification", West Sussex, UK, John Wiley \& Sons, Ltd., 2006.

[19] M. Quddus, W. Ochieng, and R. Noland, "Current Map-Matching Algorithms for Transport Applications: State-Of-The Art and Future Research Directions", Transportation Research Part C, Vol. 15, pp. 312-328, 2007.

[20] N. Velaga, M. Quddus, and A. Bristow, "Developing an Enhanced Weight-Based Topological Map-Matching Algorithm for Intelligent Transport Systems", Transportation Research Part C, Vol. 17, No. 6, pp. 672683, 2009.
[21] C. Blazquez, and A. Vonderohe, "Effects of Controlling Parameters on Performance of a DecisionRule Map-Matching Algorithm", Journal of Transportation Engineering, Vol. 135, No.12, pp. 966-973, 2009.

[22] J. Greenfeld, "Matching GPS Observations to Locations on a Digital Map", in 81st Annual Meeting of the Transportation Research Board, Washington D.C., 2002 